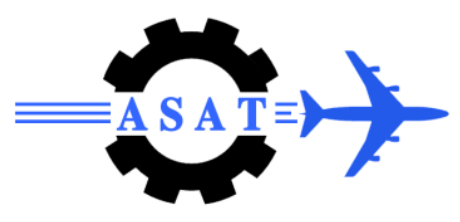

\title{
Analysis of Radar Doppler Echoes from Various Ground Moving Targets
}

\author{
Milenko S. Andrić*, Boban P. Bondžulić ${ }^{\dagger}$, Dimitrije M. Bujakovićł and Srđan T. Mitrović ${ }^{\S}$
}

\begin{abstract}
The main tasks of ground surveillance radars for security and perimeter protection are detection and classification of moving ground targets. In typical radar systems, target detection is fully automated, but the target classification requires human involvement. In this paper, we consider received radar echoes data of ground moving targets, and corresponding signals in time - frequency domain using spectrograms and cepstrum. The objective of the paper is to identify and validate the intrinsic features characterizing the different classes of targets, and subsequently extract salient features for classification. We will show examples on Radar Echoes Database. This database, named RadEch Database, cointaines radar echoes that are collected in controlled test enviroments at the premises of Military Academy - Republic of Serbia. Database purpose is to enable reproducible research results in the field of classification of ground moving targets (pattern recognition).
\end{abstract}

Keywords: Cepstrum, classification, Doppler signature, feature extraction, radar echoes database, spectrogram.

\section{Introduction}

The main tasks of ground surveillance radars for security and perimeter protection are detection and classification of ground moving targets. Many current radar-based classification systems employ some type of Doppler or Fourier-based processing, followed by spectrogram and gait analysis to classify detected targets.

The Doppler phenomenon describes the shift in the center frequency of an incident waveform due to the target motion with respect to the radar [1].

Several authors in their studies proved that spectrogram-based features could be used for discrimination purposes either between humans and other moving objects or between different persons [2]-[6]. Human spectrograms can be used to reveal information on the human's behavior and to determine features about the human target being observed, such as size, gender, action, and speed, too.

\footnotetext{
"Assistant professor, Military Academy, Department of military electrical engineering, Generala Pavla Jurisica Sturma 33, Belgrade, Serbia/asmilenko@ beotel.net .

${ }^{\dagger}$ Assistant, Military academy, Department of telecommunications and informatics, Generala Pavla Jurisica Sturma 33, Belgrade, Serbia/bondzulici@ yahoo.com .

* Assistant, Military academy, Department of telecommunications and informatics, Generala Pavla Jurisica Sturma 33, Belgrade, Serbia/dimitrije.bujakovic@ @a.mod.gov.rs .

$\S$ Assistant professor, Military academy, Department of military electrical engineering, Generala Pavla Jurisica Sturma 33, Belgrade, Serbia/srdjan.mitrovic@va.mod.gov.rs .
} 
Research done by Geisheimer and others [2] had shown that the human spectrogram is the sum of Doppler shifted signals reflected from the various parts of the moving body. Using Short Time Fourier Transform (STFT) and the chirplet transform, they extracted various parameters of the human gait from the signal. Research done by van Dorp and others [3] had shown that the radar Doppler signatures, observed in the spectrogram, give detailed information about the movements of the human body parts. The authors focused on the extraction of parameters and described a method for estimating human walking parameters from radar measurements. The application of a continuous-wave radar for the detection and classification of people based on their motion has been demonstrated in [4]. Spectral analysis of the output from the radar using a sequence of STFTs was used to extract to identify some key features of the human walking motion, and to differentiate humans from dogs. Using human gait analysis Greneker [5] designed and tested a suicide bomber detection system based on variations in the spectrogram caused by the presence of a bomb. Authors in [6] used the wavelet transform with time-frequency analysis to extract Doppler features from radar signal returns of helicopter and human targets.

A target classification algorithms using Doppler signature were presented in [7]-[11]. In [7] a Hidden Markov Model (HMM) classifier was implemented for classification between three classes of targets: personnel, tracked vehicles and wheeled vehicles. A fuzzy logic approach to the automatic classification was presented in [8]. Bilik et al [9] developed a Greedy Gaussian Mixture Model (GMM) based classification technique, applicable in classification for low resolution ground surveillance radars. The problem of classification between a walking person, pair of walking persons and slowly moving vehicle was studied in [10]. Time varying velocities and bio-mechanical human locomotion models they used for target classification. A cepstrum-based analysis method for radar target identification is proposed in [11]. The traditional nearest-neighbor classifier is used as a classifier.

At first glance, spectrogram- and cepstrum-based features seem like a promising solution for classification problems. However, the applicability and performances of these features sometimes were not tested in the context of practical systems [12]. Therefore, extensive experiments with various scenarios were carried out in order to obtain a radar echoes database (different targets and environments). In order to identify and validate the main features of the various target classes the STFT and cepstrum analysis are performed.

The remainder of the paper is organized as follows. Section 2 describes the radar echoes database which was obtained with ground surveillance radar. Sections 3 and 4 present the spectral analysis of radar Doppler signal with spectrogram and cepstrum. Section 5 presents human motion signatures in Doppler signal. Finally, concluding remarks are given in Section 6.

\section{Radar Echoes Database}

The database was obtained using records of a short range ground surveillance radar. This radar is coherent and has from the outset possessed a so called audio output which can be used by the operator to classify targets. When a RF signal is incident on a target moving towards or away from the radar, the signals reflected from the various components of the target will have a Doppler shift that is proportional to the velocity of those components.

The radar operates in the Ku-band and for this carrier frequency the Doppler frequencies lie within the audio band, being of the order of a kilohertz, and so can be presented as an audio tone to the radar operator, via headphones. When listening to this tone, it was noted that 
ground moving targets produced a very distinct and characteristic sound that, upon hearing only a few times, one learns to easily recognize.

The sensor, used in database collection, is a $16.8 \mathrm{GHz}$ ground surveillance pulse-Doppler radar. The radar parameters are: average power $-5 \mathrm{~mW}$, pulse width $-15 \mu \mathrm{sec}$, average range resolution $-150 \mathrm{~m}$, elevation resolution $-7.5^{\circ}$, and azimuth resolution $-5^{\circ}$.

For the recording procedure, the target was detected and tracked automatically by the radar, allowing continuous target echo records. The range between the radar and the target was set to be short $(100-1000 \mathrm{~m})$. The moving targets were within the line-of-sight, in the presence of ground clutter with low vegetation and without any interference. The target motions were fully controlled. One target at a time was recorded in each scenario.

Amplitude of the raw radar data was in the range $\pm 1 \mathrm{~V}$ and the sampling frequency was $4 \mathrm{kHz}$. Audio signal from radar was connected to laptop sound card microphone input. The radar's baseband audio signal was recorded onto a laptop, where the data was then saved as digitized WAV files. This allowed for the data to be easily processed using MATLAB ${ }^{\mathrm{TM}}$.

The database contains .MAT files of records of radar echoes from various targets. MAT-files, the data file format MATLAB ${ }^{\mathrm{TM}}$ software uses for saving data to disk, provide a convenient mechanism for moving data between platforms and for importing and exporting data to stand alone MATLAB applications.

The large database of the raw real audio Doppler signals was created through more than 80 different scenarios. At least $20 \mathrm{~s}$ of each scenario was recorded. In total, the database of the collected real data for each target class contained 453 records of $4 \mathrm{~s}$ duration.

The targets were recorded in two different environments. The first environment is the road of $4 \mathrm{~m}$ width, and $800 \mathrm{~m}$ length (Fig. 1(a)). The second environment is the rough terrain, with barriers (slews, woods), and with small vegetation (Fig. 1(b)).

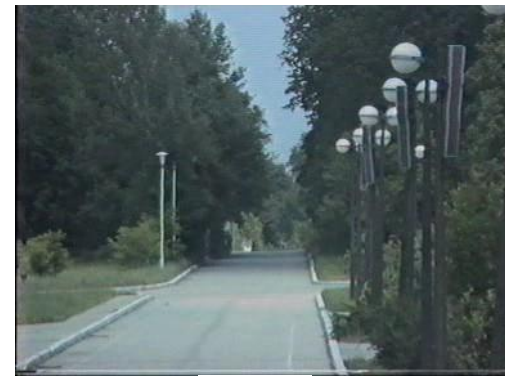

(a)

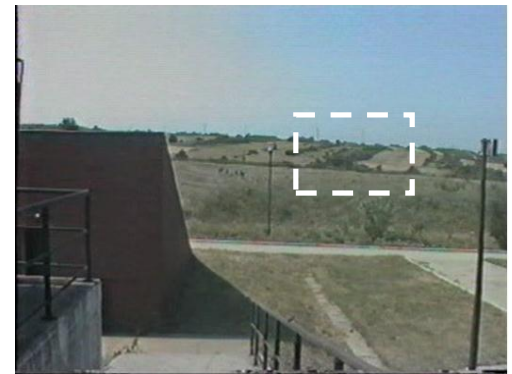

(b)

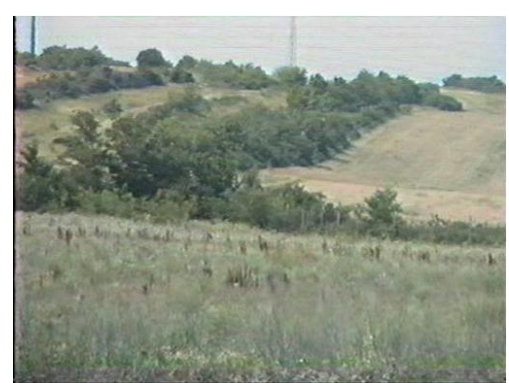

(c)

Fig. 1. Environments of recorded scenarios: (a) the road, (b) the rough terrain, and (c) enlarged part of Fig. 1(b).

Targets from the following classes were recorded:

1. Person and group of persons - combinations of the following cases were represented in the collected database:

a. Number of persons: 1,3 or more.

b. Motion: crawling, normal walking, and running.

c. Synchronous / asynchronous motion of persons in a group.

d. Pedestrian, soldier, group of persons, group of soldiers.

e. Go away - from the radar (otherwise toward the radar). 


\section{Vehicle:}

a. Wheeled / Truck vehicle.

b. Speed of motion: normal $(20-30 \mathrm{~km} / \mathrm{h})$ and fast $(30-60 \mathrm{~km} / \mathrm{h})$.

3. Vegetation clutter (trees, bush).

A database of the Doppler signatures of ground moving objects was collected along with a video camera. The system was employed at the Military Technical Institute. Data was collected on two consecutive days. A standard VHS videotape was used to record the video image from the camera. This provided simultaneous recording of the Doppler signatures and imagery of the scene. Having the video available when recording the data files allowed for the targets to be separated into different categories such as male or female, single person or groups of persons, and approaching or receding.

Detailed description of database can be found in [13], and database is freely available for download at [14].

\section{Spectral Analysis of Audio Doppler Signal}

The basic idea of the Fourier transform is that any arbitrary signal (of time, for instance) can be decomposed into a set of sinusoids of different frequencies. While the Fourier transform is a very useful concept for stationary signals, many signals encountered in real-world situations have frequency contents that change over time. In this case, it is not always best to use simple sinusoids as basis functions and characterize a signal by its frequency spectrum. Joint timefrequency transforms were developed for the purpose of characterizing the time-varying frequency content of a signal. The best-known time-frequency representation of a time signal is known as the short-time Fourier transform (STFT). It is basically a moving window Fourier transform. By examining the frequency content of the signal as the time window is moved, a 2D time-frequency distribution called the spectrogram is generated [15].

The Fourier transform representation of a discrete-time signal $x[n]$ is given by the equation:

$$
X\left(e^{j \omega}\right)=\sum_{n=-\infty}^{\infty} x[n] e^{-j \omega n},
$$

where $\omega$ is the digital frequency.

The most standard approach to analyze a signal with time-varying frequency content is to split the time-domain signal into many segments, and then take the Fourier transform of each segment. This is known as the STFT operation and is defined as:

$$
X_{n}\left(e^{j \omega}\right)=\sum_{m=-\infty}^{\infty} w[n-m] x[m] e^{-j \omega m} .
$$

In Eq. (2), $w[n-m]$ is a real window sequence which determines the portion of the input signal that receives emphasis at a particular time index, $n$. The time dependent Fourier transform is clearly a function of two variables: the time index, $n$, which is discrete, and the frequency variable $\omega$, which is continuous.

Operation (2) differs from the Fourier transform (1) only by the presence of a window function $w$. By the translation of the window as a function of time $n$, resulting in a 2D joint time-frequency representation $X_{n}\left(e^{j \omega}\right)$ of the original time signal $x[n]$. The magnitude display $\left|X_{n}\left(e^{j \omega}\right)\right|$ is called the spectrogram of the signal. It shows how the frequency spectrum (i.e., one vertical column of the spectrogram) varies as a function of the horizontal time axis.

Figures 2(a)-2(f) illustrate the Doppler spectrograms of the target classes: crawling person, walking person, running person, walking group, running group, and light wheeled vehicle. The Doppler frequency is displayed on the vertical axis and time on the horizontal. The amplitude of the reflected signal is color coded with red being the highest intensity and blue 
the lowest. These spectrograms clearly show a difference between target class characteristics, which can be used for classification.



(a)



(d)

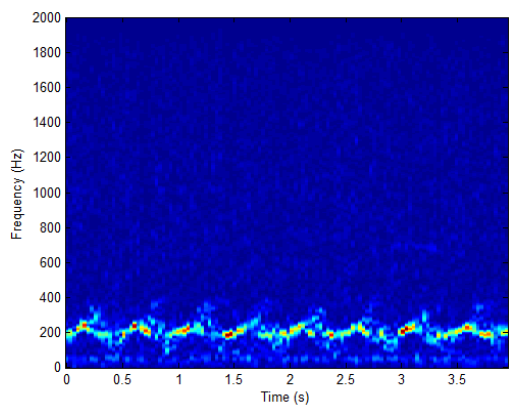

(b)

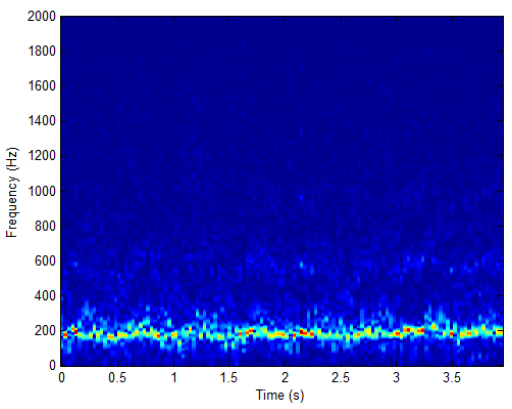

(e)

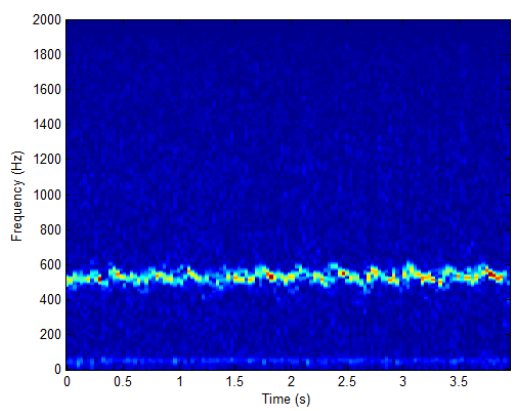

(c)

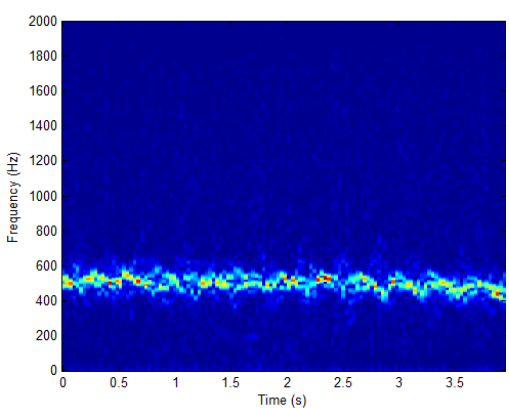

(f)

Fig. 2. Spectrograms of radar echo target samples: (a) person crawling, (b) person walking, (c) person running, (d) vehicle, (e) group of persons walking, and (f) group of persons running.

A crawling person spectrogram, Fig. 2(a), is periodic, but with pauses due to type of motion. When humans walk, the motion of various components of the body including the torso, arms, and legs produce a very characteristic Doppler signature, Fig. 2(b). Doppler spectrogram from a running person is presented in Fig. 2(c). We can see a specific person signature which is characterized with component which oscillates in frequency (characteristic quasi-periodic signal).

Spectrogram from light-wheeled vehicle (car) is shown on Fig. 2(d). The signature from moving wheeled vehicle has one dominant spectral line at Doppler frequency and narrow band of spectral components around central Doppler frequency because the wheeled vehicle is compact target without moving subreflectors.

The comparative analysis of spectral characteristics of these three target classes shows the difference in central Doppler frequency and the width of spectral line around central Doppler frequency.

The presence of several people simultaneously in the radar field of sight, irrespective of walking/running motion type, involve interferences. Thus, the spectral widths around central Doppler frequency increase (Fig. 2(e), and Fig. 2(f)).

As a conclusion, the band of spectral components around central Doppler frequency is the least in the case of wheeled vehicle in comparison with a walking and running person case. In the case consequently when the central Doppler frequencies of a running person and wheeled vehicle with low velocities are similar, may be used to resolve this type of classification conflict the width of spectral band around the central Doppler frequencies.

Based on spectral analysis of Doppler signal by spectrogram, central Doppler frequency and width of spectral band around it are possible features for input fuzzy variables. 


\section{Analysis of Doppler Signal Based on Real Cepstrum}

A cepstrum is the result of taking the inverse Fourier transform (FT) of the log spectrum as if it were a signal. Its name was derived by reversing the first four letters of spectrum. There is a complex cepstrum, a real cepstrum, a power cepstrum, and phase cepstrum.

The real cepstrum $c[n]$ of a discrete-time signal $x[n]$ is defined as [9]:

$$
c[n]=F^{-1}\{\log |F\{x[n]\}|\},
$$

where $F[\cdot]$, and $F^{-1}[\cdot]$ are the discrete Fourier and the inverse Fourier transforms, respectively.

There are many ways to calculate the cepstrum. Some of them need a phase-unwrapping algorithm, others do not. Operations on cepstra are labeled quefrency alanysis, liftering, or cepstral analysis.

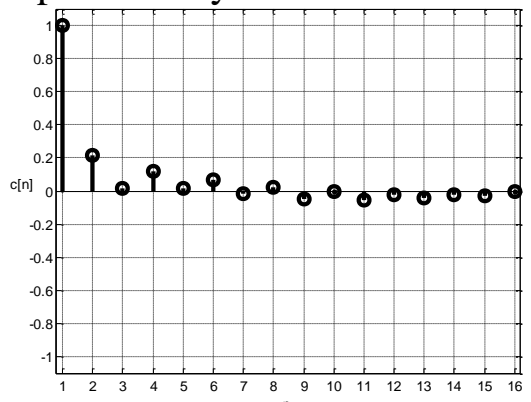

(a)

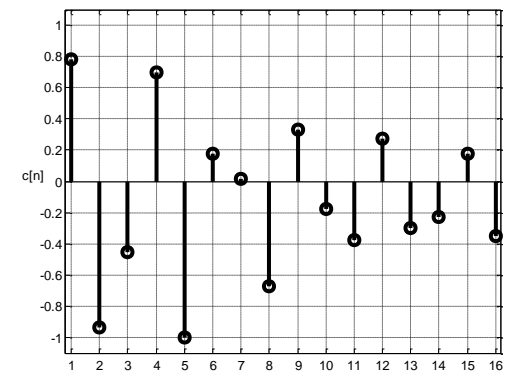

(d)

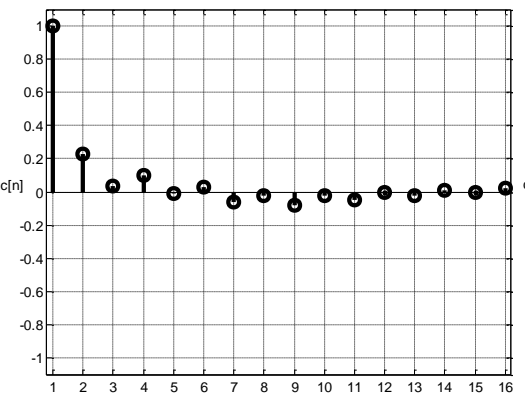

(b)



(e)

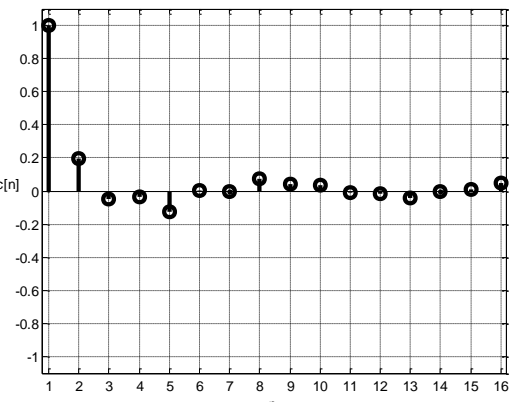

(c)

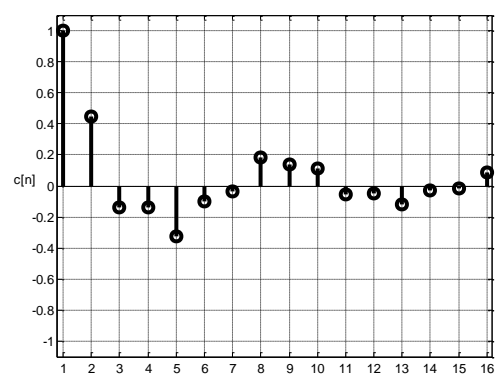

(f)

Fig. 3. Real cepstrums of radar echo target samples: (a) person crawling, (b) person walking, (c) person running, (d) vehicle, (e) group of persons walking, and (f) group of persons running.

In [16], [17] cepstrum was used in speech signal representation and further for speaker recognition application. The classification features used in [10] are cepstrum coefficients. In [11] authors used cepstrum and real cepstrum for radar target identification from the three classes of targets: person, tank, and bicycle. They obtained the average identification accuracy of $70.7 \%$, and concluded that the complex cepstrum and cepstrum are better for radar target identification than spectrum. In [11] authors used normalized amplitude characteristics of radar target function $H\left(e^{j \omega}\right)$ for classification, which is given by the following equation:

$$
H\left(e^{j \omega}\right)=\exp (F\{c[n]\})
$$

The real cepstrum is compressed in time since the energy of the real cepstrum concentrates nearby origin, and due that fact we used a low-time window which length is sixteen. 
In Fig. 3 we showed a normalized real cepstrums of analyzed radar echo target samples. Samples are same as samples used for spectrogram calculation (Fig. 2). Normalized amplitude characteristics, $H\left(e^{j \omega}\right)$, of radar targets are showed in Fig. 4.

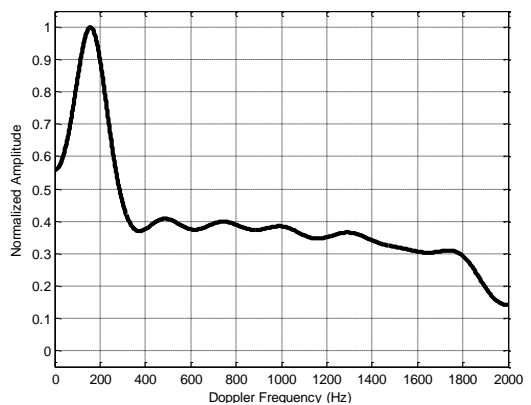

(a)

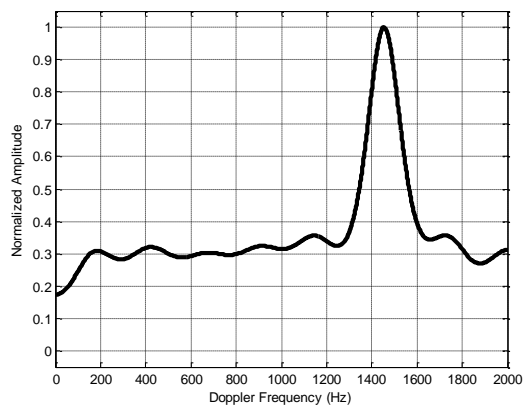

(d)

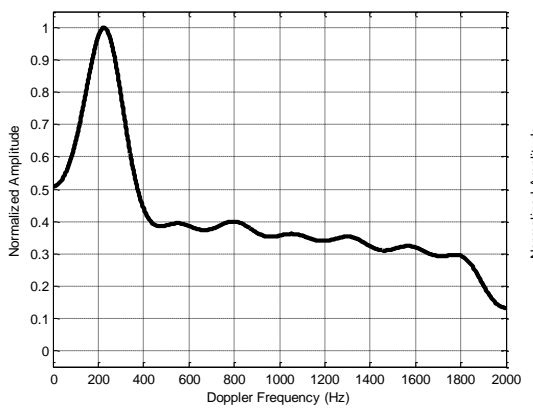

(b)

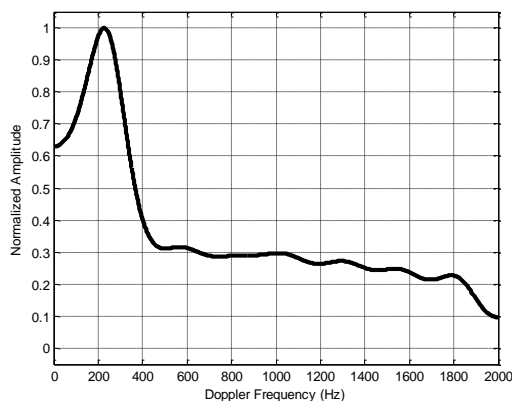

(e)

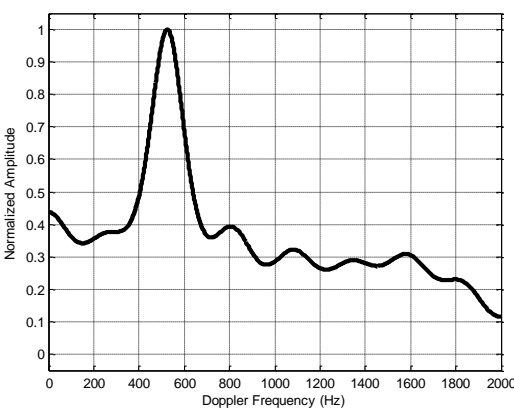

(c)



(f)

Fig. 4. Normalized amplitude characteristics of radar targets: (a) person crawling, (b) person walking, (c) person running, (d) vehicle, (e) group of persons walking, and (f) group of persons running.

From Fig. 4 we can see that there are differences between central Doppler frequency.

Cepstrum of radar signals gives an information about whole sequence, and do not give information about sequence changes during time. Because of that, in the rest of paper, we used only spectrogram-based analysis of Doppler signal. This can be overreach by using a "running cepstrum" algorithm.

\section{Human Motion Signatures in Doppler Signal}

Human locomotion consists of a complex movement of various parts of the body [18]. When a human moves, the different parts of his body (torso, arms, legs) have a particular motion that produces characteristic Doppler signatures (see Fig. 2(b)). The spectral amplitude corresponds to the radar cross section (RCS) of the moving parts. The main contribution comes from the torso. The motion of arms and legs induces modulation on the returned radar signal and generates sidebands about the Doppler frequency, referred to as micro-Doppler signatures. They can provide valuable information about the structure of the moving parts and may be used for classification purposes [19].

Since the motion of the legs and arms of a walking person is periodic, Otero in [4] used the Fourier transform to extract basic information such as the cadence frequency from the spectral image, which is the step or leg swing rate. For each Doppler bin on the spectrogram vertical axis an FFT was applied over the entire time frame. The result is that the vertical scale is preserved and the horizontal time axis is transformed to the frequency domain. 
An example of the radar echo spectrogram for a person walking is shown in Fig. 5(a). Notice the significant spread of the Doppler component caused by the varying motion of the appendages. A sample cadence frequency plot is shown below in Fig. 5(b).

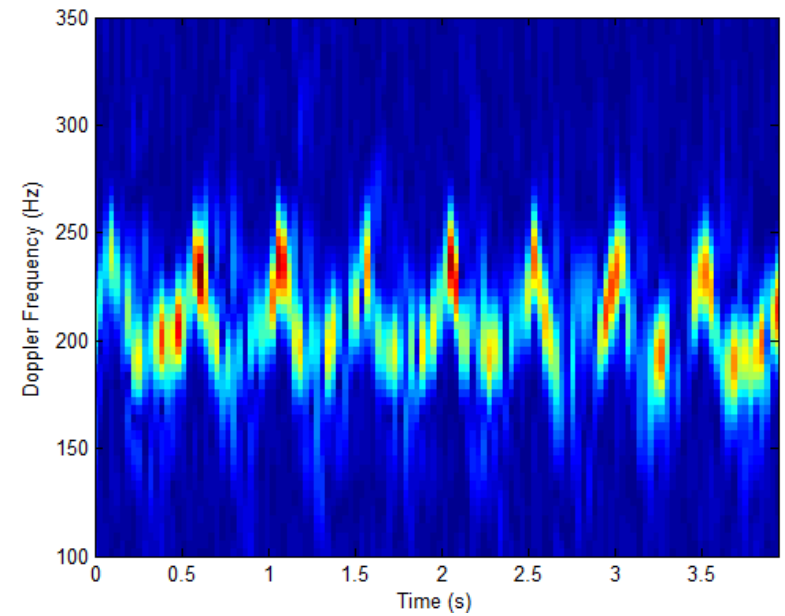

(a)

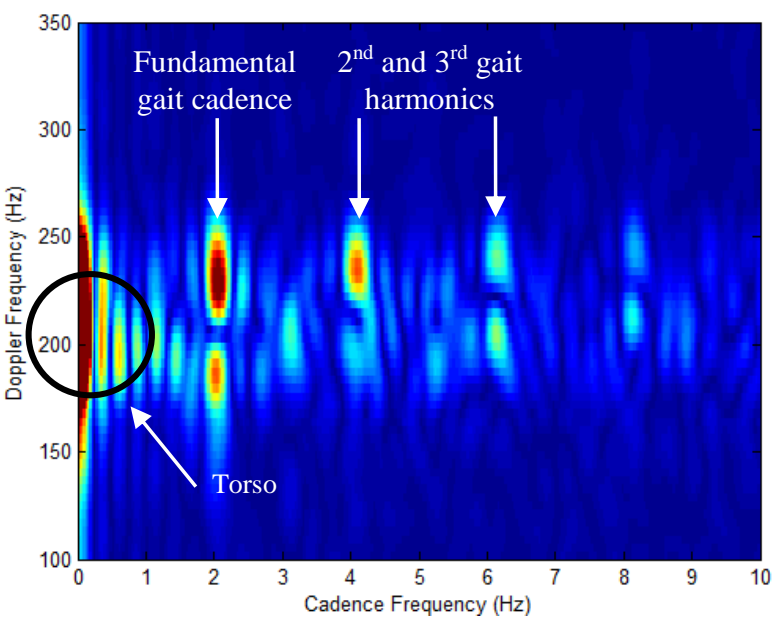

(b)

Fig. 5. (a) Spectrogram of a person walking, and (b) cadence frequency plot of Fig. 5(a).

The cadence frequency plot highlights the periodic signals present in the spectrogram. The two dominant parameters which characterize the human gait (mean velocity and step rate) can be deduced from suitable portions of the cadence plot. The peak at about zero cadence frequency corresponds to the motion of the torso, with the value of the Doppler frequency $\left(f_{\text {Dtorso }}\right)$. The next peak corresponds to the fundamental frequency, $f_{m}$. Subsequent peaks result from the motion of the other appendages, such as the arms and legs [4],[12].

With the Doppler formula,

$$
v=\frac{\lambda \cdot f_{\text {Dtorso }}}{2}
$$

where $\lambda$ is the sensor wavelength, it can be seen that the walking speed $v$ is proportional to the measured frequency.

By then dividing the walking speed by the cadence frequency yields the length of the stride, $l_{s}$,

$$
l_{s}=\frac{v}{f_{m}},
$$

In the example of Fig. 5(b) the torso component has a Doppler frequency of about $210 \mathrm{~Hz}$ indicating the person was moving with a speed, using (5), of about $1.87 \mathrm{~m} / \mathrm{s}$. Due to the spectral spread this curve will include errors if the detected velocity is not identical to the mean velocity of the torso. The modulation of the legs has a fundamental cadence frequency, $f_{m}$, of about $2 \mathrm{~Hz}$ and the stride length, using (6), is $0.94 \mathrm{~m}$. Because of known dependencies between step frequency and velocity for human gait, it is expected to be useful to combine these both features for target classification.

Otero in [4] was considered the amplitudes of the cadence plot peaks to be indicative of RCS. The RCS of the appendages he determined by summing the amplidudes of the peaks of the fundamental and $2^{\text {nd }}$ and $3^{\text {rd }}$ harmonics that are associated with the periodic motion of the 
arms and legs. This is then divided by the amplitude of the torso peak in the spectrum. This feature is reffered to as the appendage/torso ratio.

To obtain an valid estimate values of fundamental cadence frequency and appendage/torso ratio noise and clutter have detrimental effect. Clutter is due to unwanted reflections off of objects in the environment that are not targets, such as trees, asphalt roads, buildings, and multi-path target reflections.

An example of the radar echo spectrograms and appropriate cadence frequency plots for a person and group of persons classes are shown in Fig. 6 and Fig. 7.



(a)



(b)

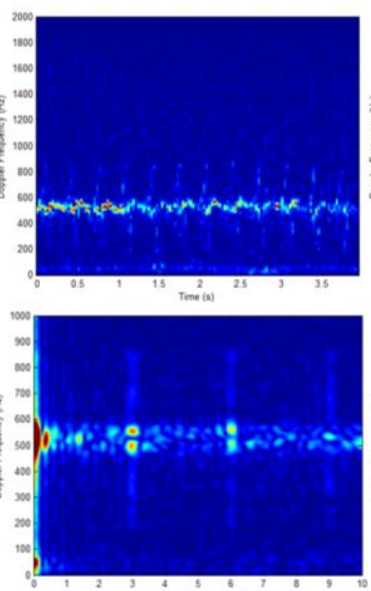

(c)

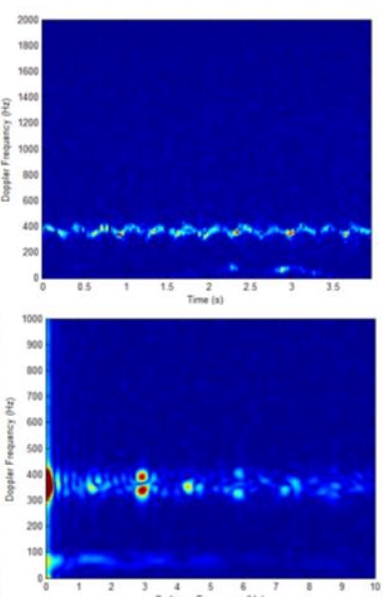

(d)

Fig. 6. Spectrograms (upper row) and appropriate cadence frequency plots (bottom row) for a person target class: (a) walking ot the road, (b) walking on the bush, (c) running on the road, and (d) running on the bush.

Fig. 6 shows that ambient (road and bush) has a great importance in appendage/torso ratio estimation. If the leg swings are hidden with vegetation, higher order harmonics are reduced at cadence frequency plots (see Fig. 6(b) and Fig. 6(d)). Moreover, valid fundamental cadence frequency can be estimate for analyzed situations.

Unfortunately, estimates of these parameters derived from the cadence frequency plot tend to be quite unreliable in the presence of several persons simultaneously in the radar field of sight (see Fig. 7). Fig. 7(a) and Fig. 7(b) shows that in some cases fundamental cadence frequency peak appear since group of people have synchronous motions.

Finally, fundamental cadence frequency provides additional information to differentiate person class from the others. The length of the time window must be chosen to provide enough gait cycles to resolve the cadence frequency for a typical walking/running person.

\section{Conclusion}

The application of a pulse-Doppler radar for the classification of moving ground targets has been demonstrated. The spectral analysis conducted in this paper is used to extract very basic information that could be used for classification; thus, the observations remained as suggestions requiring further research.

Further work would be needed to create a multi-class classifier that can discriminate different target classes. 


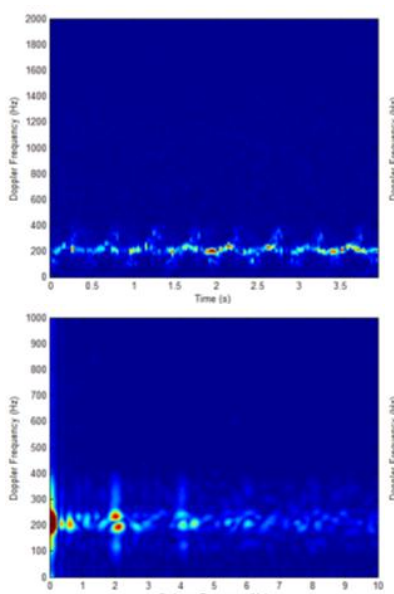

(a)

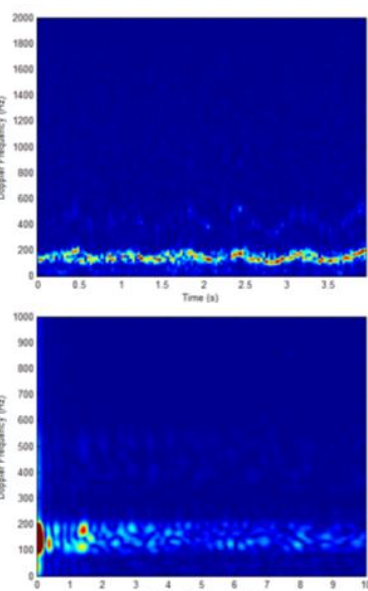

(b)

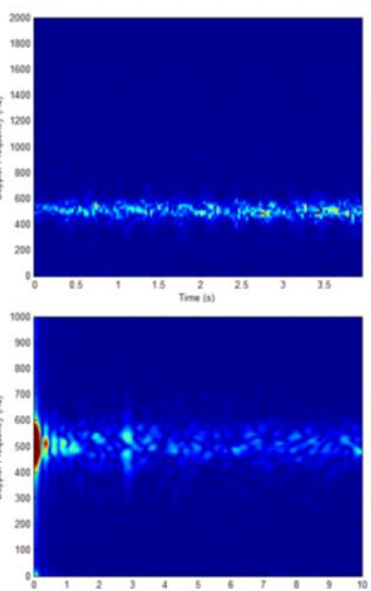

(c)

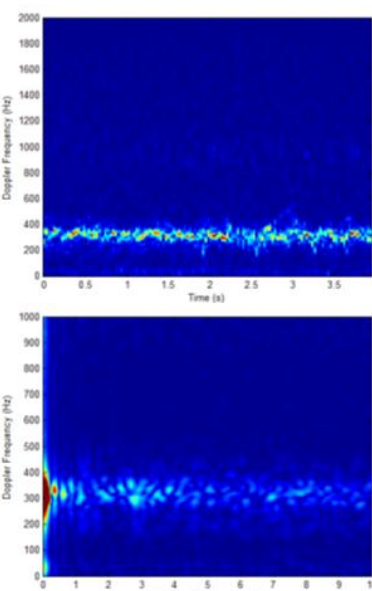

(d)

Fig. 7. Spectrograms (upper row) and appropriate cadence frequency plots (bottom row) for a group of persons target class: (a) walking on the road, (b) walking on the bush, (c) running on the road, and (d) running on the bush.

\section{References}

[1] Avci, E., Turkoglu, I., and Poyraz, M., "A new approach based on wavelet nero genetic network for automatic target recognition with X-band Doppler radar," Journal of Electrical \& Electronics Engineering, Vol. 6, No. 2, 2006.

[2] Geisheimer, J.L., Marshall, W.S., and Greneker, E., "A Continuous-Wave Radar for Gait Analysis," in Proc. $35^{\text {th }}$ Asilomar Conference on Signals, Systems, and Computers, Vol. 1, pp. 834-838, 2001.

[3] van Dorp, P., and Groen, F.C.A., "Human Walking Estimation with Radar," IEE Proc.Radar Sonar Navig., Vol. 150, No. 5, 2003.

[4] Otero, M., "Application of a continuous wave radar for human gait recognition," in Proc. of SPIE, Vol. 5809, pp.538-548, 2005.

[5] Greneker, G., "Very low cost stand-off suicide bomber detection system using human gait analysis to screen potential bomb carrying individuals," in Proc. of SPIE, Vol. 5788, pp. 46-56, 2005.

[6] Thayaparan, T., Abrol, S., Riseborough, E., Stankovic, Lj., Lamothe, D., and Duff, G., "Analysis of radar micro-Doppler signatures from experimental helicopter and human data," IET Radar Sonar Navig., Vol. 1, No. 4, pp. 289-299, 2007.

[7] Jahangir, M., Ponting, K.M., and O'Loghlen, J.W., "Robust Doppler classification technique based on Hidden Markov models," IEE Proc.-Radar Sonar Navig., Vol. 150, No. 1, pp. 33-36, Feb. 2003.

[8] Andrić, M., Đurović, Ž., and Zrnić, B., "Ground Surveillance Radar Target Classification Based on Fuzzy Logic Approach," in Proc. of the Int. Conference on Computer as a Tool, Vol. 2, pp. 1390-1392, 2005.

[9] Bilik, I., Tabrikian, J., and Cohen, A., "GMM-based target classification for ground surveillance Doppler radar," IEEE Trans. Aerospace and Electronic Systems, Vol. 42, pp. 267-278, 2006.

[10] Bilik, I., and Tabrikian, J., "Radar target classification using Doppler signatures of human locomotion models," IEEE Trans. Aerospace and Electronic Systems, Vol. 43, No. 4, pp. 1510-1522, 2007. 
[11] De-Quan, Z., and Yan-Qing, G., "Study of Radar Target Identification Based on Cepstrum and Complex Cepstrum," International Symposium on Microwave, Antennas, Propagation and EMC Technologies for Wireless Communications, 2007.

[12] Gurbuz, S.Z., Melvin, W.L., and Williams, D.B., "Detection and Identification of Human Targets in Radar Data," in Proc. of SPIE Defence and Security Symposium, Orlando, Fl, USA, April 2007.

[13] Andric, M., Bondzulic, B., and Zrnic, B., "The Database of Radar Echoes from Various Targets with Spectral Analysis," 10th Symposium on Neural Network Applications in Electrical Engineering - NEUREL, Faculty of Electrical Engineering, University of Belgrade, Serbia, September 23-25, 2010.

[14] The Database of Radar Echoes from Various Targets [Online]. http://cid3aaf3e18829259c0.skydrive.live.com/home.aspx, 2010.

[15] Chen, V.C., Ling, H., Time-frequency transforms for radar imaging and signal analysis, Artech House, Inc., 2002, Chapter 2, pp. 25-46.

[16] Rabiner, L.R., Schafer, R.W., Digital Processing of Speech Signals, Artech House, 1978, Chapter 7, pp 355 - 395.

[17] Rabiner, R., Schafer, R.W., Theory and Application of Digital Speech Processing, Preliminary Edition, 2009, Chapter 7, pp 423 - 475.

[18] Hornsteiner, C., and Detlefsen, J., "Characterisation of human gait using a continuouswave radar at $24 \mathrm{GHz}$," Advances in Radio Science, Vol. 6, pp. 67-70, 2008.

[19] Vignaud, L., Ghaleb, A., Le Kernec, J., and Nicolas, J.-M., "Radar high resolution range \& micro-doppler analysis of human motions," International Radar Conference RADAR, 2009. 